\title{
Temperature measurements in sooting counterflow diffusion flames using laser-induced fluorescence of flame-produced nitric oxide
}

\author{
Journal Article \\ Author(s): \\ Denisov, Alexey; Jansohn, Peter; Colmegna, G. \\ Publication date: \\ 2014-08 \\ Permanent link: \\ https://doi.org/10.3929/ethz-b-000088130
}

Rights / license:

In Copyright - Non-Commercial Use Permitted

Originally published in:

Applied Physics B 116(2), https://doi.org/10.1007/s00340-013-5697-6 


\title{
Temperature measurements in sooting counterflow diffusion flames using laser-induced fluorescence of flame-produced nitric oxide
}

\author{
A. Denisov $\cdot$ G. Colmegna $\cdot$ P. Jansohn
}

Received: 14 May 2013/Accepted: 16 October 2013/Published online: 25 October 2013

(C) Springer-Verlag Berlin Heidelberg 2013

\begin{abstract}
Laser-based diagnostic methods are often used for non-intrusive studies of delicate processes of soot formation. When soot particles are heated by the laser pulse, their size distribution can be estimated from the cooling rate, provided that the local gas temperature is known. However, strong light absorption, scattering and fluorescence in sooting environment hinder non-intrusive laserbased temperature measurements. Methods based on fitting of laser-induced fluorescence spectra work well in stationary flames but usually require temperature tracer seeded into the flame. We have shown that in counterflow diffusion flames, often used for soot-formation studies, enough nitric oxide is produced for two-dimensional temperature imaging. Measured temperature profiles agree very well with chemical kinetic calculations for a variety of fuels if laser intensity is reduced to keep NO excitation in the linear regime. Gas composition affects line shapes at temperatures below $600 \mathrm{~K}$ and should be taken into account for accurate measurements.
\end{abstract}

\section{Introduction}

Spatially-resolved temperature measurements are very useful in combustion diagnostics. For example, timeresolved laser-induced incandescence sizes soot particles based on their cooling rates and requires the local gas temperature to be known with an accuracy of at least $50 \mathrm{~K}$

\footnotetext{
A. Denisov $(\bowtie) \cdot$ P. Jansohn

Paul Scherrer Institute (PSI), 5232 Villigen, Switzerland

e-mail: alexey.denisov@psi.ch

G. Colmegna

Methanol Casale, Via G. Pocobelli 6, 6900 Lugano, Switzerland
}

[1]. However, strong light absorption and scattering as well as large number of fluorescing species in fuel-rich sooting environments pose serious challenges for laser-based methods. For example, strong scattering of laser light by soot particles prevents detection of much weaker Rayleigh scattering from gas molecules. Interference from soot can be suppressed using a narrow-band filter, but determining gas temperature from the remaining part of molecular scattering [2] requires precise modeling of Rayleigh lineshapes and knowledge of local gas composition.

Vibrational coherent anti-Stokes Raman spectroscopy, or CARS, is a well-established method for accurate temperature measurements in flames [3]. In sooting conditions, however, the traditional configuration using $532 \mathrm{~nm}$ for both pump beams is complicated by $\mathrm{C}_{2}$ absorption and emission that coincide spectrally with the generated antiStokes beam [4]. Approaches that avoid $\mathrm{C}_{2}$ interference, such as dual-pump CARS [5], are less preferred due to increased complexity.

Methods based on laser-induced fluorescence (LIF) are attractive because of the relatively simple experimental arrangement that provides the possibility of two-dimensional temperature measurement. Two-line atomic fluorescence was proposed as a method, particularly suited to fuel-rich flames [6]. Potentially capable of instantaneous temperature measurements, it is hindered by poor signal-tonoise ratio due to the low concentration of tracer atoms. Strong atomic transitions are easily saturated at modest laser intensity, and the nonlinear excitation regime should be modeled to allow for higher laser energies [7]. Besides atomic tracers, a significant amount of solvent (3 vol\%) is introduced into the flame, which may affect soot-formation processes.

Two-line temperature measurements in flames are also done with molecular LIF tracers-mainly $\mathrm{OH}$ [8] and $\mathrm{NO}$ 
[9]. Nitric oxide has an advantage of chemical stability at both low and high temperatures that allows measurements throughout the flame. Also, thanks to fast redistribution of rotational energy in the excited state, the quenching rate for NO is similar for all rotational levels [10], so that relative line intensity does not depend on gas composition that varies strongly in diffusion flames.

This method cannot account for the broadband fluorescence of polycyclic aromatic hydrocarbons (PAHs) and is not suitable for sooting flames. One proposed solution [11] is to record a continuous NO LIF spectrum and fit a model to it. This method, conventionally called multiline thermometry, is less susceptible to interferences from fluorescence of other species, laser scattering and nonuniformity of laser-sheet intensity profile. It was later developed into a two-dimensional temperature imaging method and applied for studying sooting premixed [12] as well as non-sooting diffusion [13] flames at atmospheric pressure and for lean premixed flames at pressures up to 60 bar [14].

In previous studies, $\mathrm{NO}$ was seeded into the flame to increase signal-to-noise ratio, even though it is known that in fuel-rich conditions most of the seeded NO reacts within the flame zone [15]. While it is generally assumed that NO seeding does not change the flame temperature $[12,16]$, it was suggested that the addition of NO may affect fuel oxidation rate and decrease soot formation [17]. This makes NO seeding undesirable for studying soot inception and growth. On the other hand, significant amounts of NO can be produced in diffusion flames through the thermal and prompt NO mechanisms [18, 19] with quantitative LIF measurements in oxygen-enriched counterflow diffusion flames reporting peak concentrations well above $1,000 \mathrm{ppm}$ [20]. If this $\mathrm{NO}$ is distributed far enough throughout the burner, it can be used for truly non-intrusive temperature measurements in such flames.

In this work, we use flame-produced NO for multiline LIF thermometry in a sooting counterflow diffusion flame and compare the results to chemical kinetic calculations for a variety of fuels. We also discuss issues that impair the accuracy of multiline temperature measurements in diffusion flames.

\section{Experiment}

\subsection{Counterflow burner}

Opposed-jet diffusion flames are widely used in combustion studies [21] for their optical accessibility and geometric simplicity that facilitates chemical kinetic modeling. For this work, we used a new rectangular counterflow type II burner designed for counterflow premixed and diffusion flames (Fig. 1). Two identical heads oppose each other,

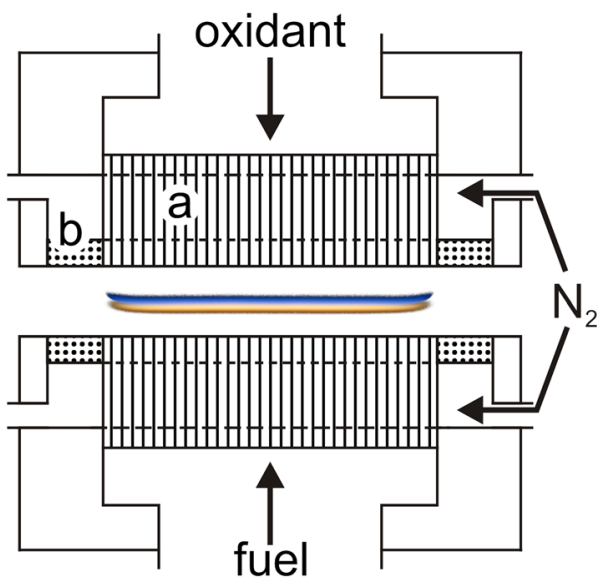

Fig. 1 A scheme of the counterflow diffusion flame burner. Fuel and oxidant pass through rectangular ceramic honeycombs $(a)$ to ensure uniform, laminar flow. The flow speed is matched by the flow of nitrogen through sintered metal plates $(b)$ that is used to prevent ignition of unburnt fuel outside of the burner

providing fuel flow from the bottom and oxidant flow from the top. The main gas channel of each head has a rectangular shape with dimensions of $6 \mathrm{~cm} \times 1 \mathrm{~cm}$. The velocity profile of the jet is made as uniform as possible using filling material in the gas channels and installing rectangular ceramic honeycombs $\left(1.4 \mathrm{~mm}^{2}\right.$ channel size $)$ at the outer sections. To prevent ignition of the unburned fuel around the burner, a flow of nitrogen is supplied through sintered metal plugs surrounding the main gas inlet sections. The flow speed of nitrogen is set to match that of the inner jet in order to minimize mixing of the two flows. Thermocouples are installed in both heads upstream of the honeycomb inserts to monitor the temperature of the flowing gases.

Flow speed of fuel and air, set equal, was varied in the range of $10-50 \mathrm{~cm} / \mathrm{s}$. A flow speed of $10 \mathrm{~cm} / \mathrm{s}$ corresponds to a Reynolds number of about 20 (referred to the equivalent diameter of the honeycomb passage) guaranteeing a laminar flow. We used a range of fuels from non-sooting methane to heavy-sooting ethylene. Oxygen content in the oxidizing flow could be varied by enriching air with oxygen.

\subsection{Laser and optical setup}

The general arrangement of the experimental setup is shown in Fig. 2. We used an Nd:YAG-pumped (Quantel YG781) dye laser (Quantel TDL90) to produce laser light with a wavelength of $572 \mathrm{~nm}$ that was frequency-doubled and then frequency-mixed with the residual Nd:YAG fundamental beam resulting in a wavelength of around $225 \mathrm{~nm}$ that was used for NO excitation. The spectral linewidth of the laser was $0.3 \mathrm{~cm}^{-1}$ (FWHM), determined by fitting NO LIF spectrum taken in a low-pressure (10 mbar) cell at room temperature. The energy of the laser 


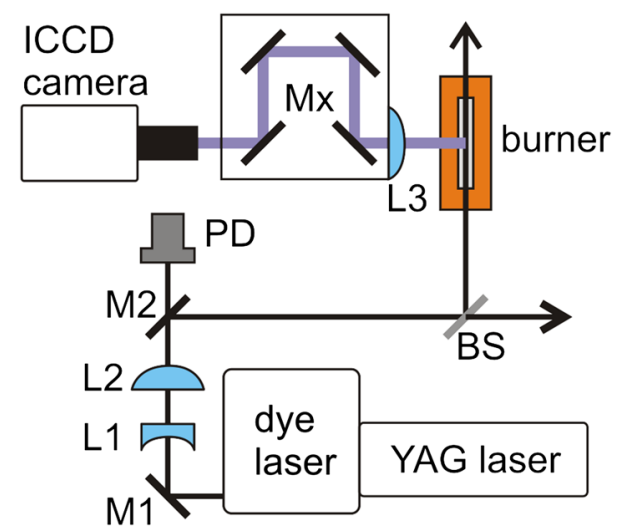

Fig. 2 Optical arrangement of the experiment, see text for the explanation of individual elements

pulse, measured by pyroelectric detector, was about $1.2 \mathrm{~mJ}$.

A combination of an expanding cylindrical lens (L1, $f=-200 \mathrm{~mm}$ ) and a collimating spherical lens (L2, $f=+300 \mathrm{~nm}$ ) placed about $100 \mathrm{~mm}$ apart formed a converging horizontal laser sheet at $0.5 \mathrm{~m}$ after L2 and then a secondary, diverging vertical sheet at about $2 \mathrm{~m}$ distance. With such an arrangement, both lenses can be placed on the optical table close to the laser and the focal position of the vertical laser sheet could be varied by adjusting the distance between lenses L1 and L2.

Particular care had to be taken to avoid LIF saturation (the influence of laser intensity on temperature fits is considered in Sect. 3.2). We attenuated the laser beam using a $33 \%$ reflecting beam splitter (BS) instead of one of the guiding mirrors and additionally reduced energy flux through the observation volume by slight defocusing of the laser sheet (FWHM of the sheet at the point of measurement was $0.7 \mathrm{~mm}$ ).

Fluorescence was collected with a spherical lens L3 $(f=+200 \mathrm{~mm})$. To discriminate against scattered light and broadband fluorescence by PAH, collected light was spectrally filtered using a combination of four dielectric mirrors reflecting in the range of 243-282 nm. Filtered light was imaged with a photographic UV lens (CERCO 95/4.1) into an image-intensified CCD camera (PCO diCamPro). The geometric resolution (calculated as ratio of the pixel count to the image length) was 32.5 pixel per $\mathrm{mm}$. For each wavelength step, the image intensifier was exposed 20 times. Relative laser pulse energy, measured with a fast UV photodiode, was used to normalize the acquired images for varying laser intensities.

\subsection{Data acquisition and processing}

Multiline thermometry is based on fitting of a continuous part of rotationally resolved spectrum, including

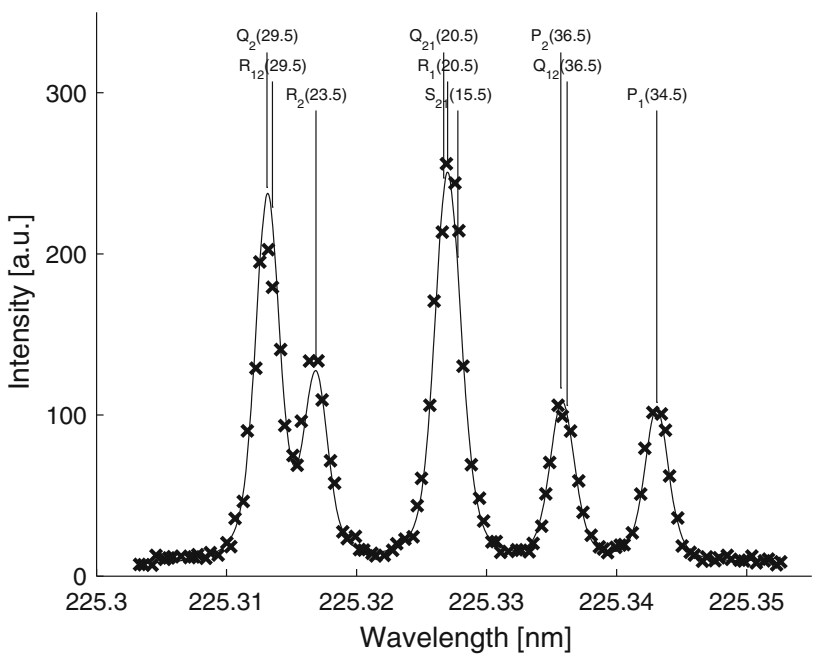

Fig. 3 Measured NO LIF intensities at the hottest part of the flame compared to the simulated rotational spectrum. Positions of rotational transitions are marked with vertical lines

nonresonant background. Including more NO lines in the spectrum improves precision of the fitted parameters but also increases the time needed to collect and process the data. To find appropriate wavelength region for multiline thermometry, we used LIFSIM [22] to simulate rotational NO LIF spectra with added random noise, fitted them and evaluated fit quality and resulting temperature values. Based on this analysis, we chose a range of $44,375-44,385 \mathrm{~cm}^{-1}$ containing nine NO rotational lines with high sensitivity to temperatures around 1,600 K (sootinception limit). With the laser wavelength scanned in steps of $0.1 \mathrm{~cm}^{-1}$, it took about $2.5 \mathrm{~min}$ to acquire $100 \mathrm{NO}$ LIF images.

This way, rotational spectrum of NO was recorded for every pixel of an image. Since the flame in our experiment was flat, we averaged a central part of the NO LIF images, corresponding to $10.8 \mathrm{~mm}$ in horizontal direction, along the horizontal axis to increase signal-to-noise ratio and reduce computational time. As LIFSIM contains all necessary constants to simulate the NO spectrum, it can also do least-squares fitting with respect to selected parameters. Figure 3 compares such fit with NO LIF spectrum taken at the hottest point of the flame (Tfit $=2,060 \mathrm{~K}$ ). Besides temperature, also intensity and baseline were fitted, the latter especially useful to account for broadband fluorescence of aromatic molecules in sooting region. Combining fit results for all points along the vertical axis, we obtained temperature profiles that are presented in this paper.

Molecular collisions affect the intensity and the shape of rotational lines, which is taken into account in the fitting procedure. Ideally, the gas composition should be known for every part of the flame, together with the collisional coefficients for every species. However, it is not practical 


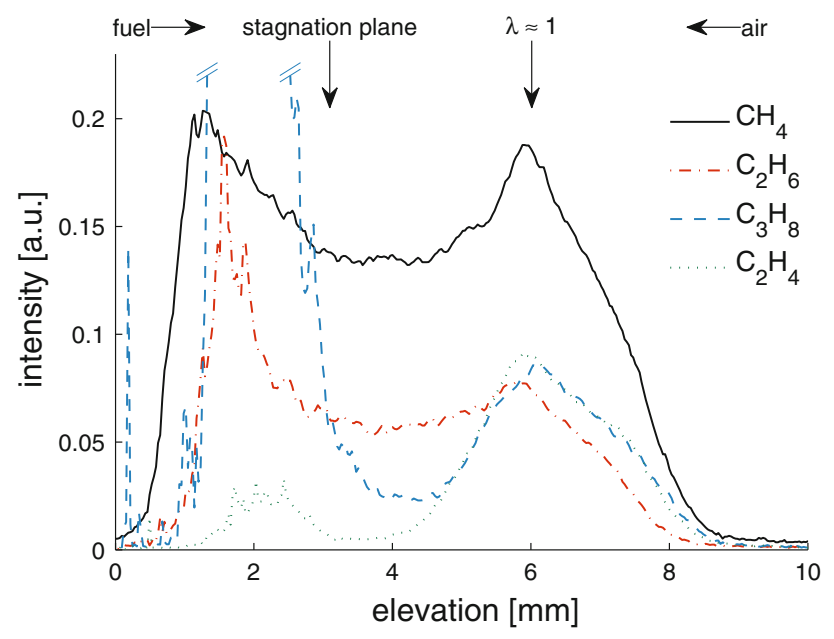

Fig. 4 Fitted intensity profiles for NO LIF measurements in flames with various fuels. Arrows at the top denote directions of fuel and air flow, as well as approximate positions of stagnation plane and hottest, stoichiometric flame zone. Gas-flow velocity was set to $10 \mathrm{~cm} / \mathrm{s}$

to calculate the gas composition for every single flame condition, and we determined that specifying nitrogen as the only collisional partner produces good results in most cases. In Sect. 4.2, we discuss the effect of this assumption on temperature fits and the difference it makes compared to using calculated detailed flame composition.

\section{Experimental considerations}

\subsection{Intensity of measured NO LIF}

The quality of the temperature fit depends on the strength of the recorded NO LIF signal that among other factors includes the intensity of the laser beam, the concentration of nitric oxide and the quenching rate of excited molecules.

Nitric oxide is primarily produced in the hottest, stoichiometric part of the flame. Due to fast rotational energy transfer (RET), NO quickly reaches thermal equilibrium with the environment so that even in this region the LIF spectrum can be fitted well assuming Boltzmann energy distribution (see Fig. 3). From this zone, NO is carried downstream toward the stagnation plane and a significant amount of NO diffuses upstream toward the burner head. As a result, NO LIF can be observed in a large part of the gap between the burner heads (see Fig. 4). At the air inlet, the signal is limited by oxidation of $\mathrm{NO}$ toward $\mathrm{NO}_{2}$ at low temperatures $\left(<1,000{ }^{\circ} \mathrm{C}\right)$, whereas at the fuel inlet the LIF intensity is high as the decrease of NO concentration is compensated by the decrease of collisional quenching rates. Saturated hydrocarbons cannot quench excited NO molecules due to their negligible electron affinity [10], which increases the fluorescence quantum yield of NO LIF

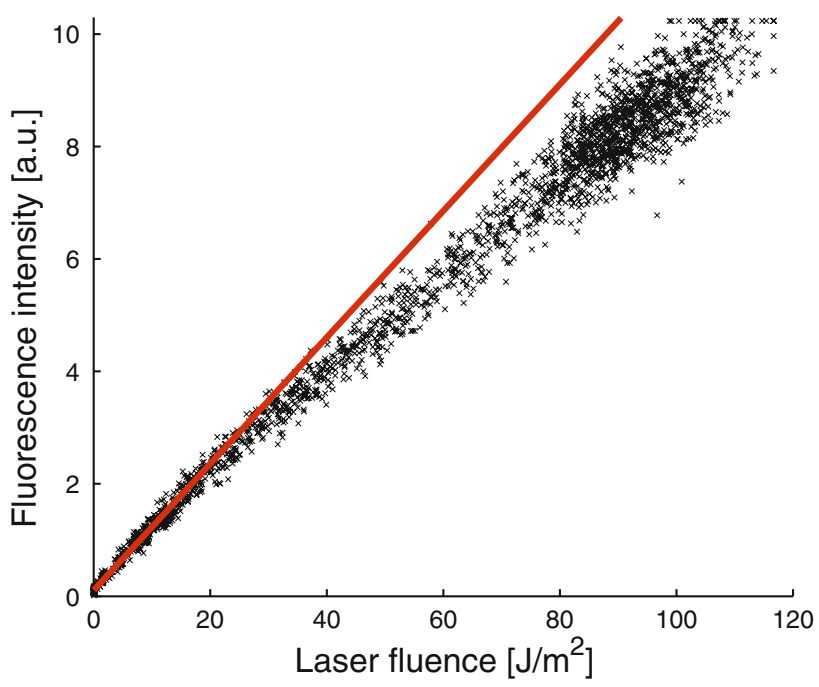

Fig. 5 Measured NO LIF intensities in a Bunsen flame deviate from linear dependence (solid line) for laser energy fluence above $20 \mathrm{~J} / \mathrm{m}^{2}$

by orders of magnitude. Closer to the burner heads, NO concentration decreases so much that reliable LIF fitting is no more possible. The temperature in these regions could not be measured.

As the laser passes along the flame front, it is absorbed by soot and its precursors. In strongly sooting conditions, using ethylene as a fuel, the observed LIF signal decreased 20 times in the region of soot formation. Polycyclic aromatic hydrocarbons (PAHs), precursors of soot, have strong absorption in the UV region, which would favor methods that use laser wavelengths in the visible region. On the other hand, broadband fluorescence in the sooting region was quite weak compared to NO LIF. We attribute it to the fact that the fluorescence of PAH molecules is located mostly at wavelengths above $300 \mathrm{~nm}$-well separated from the NO fluorescence and blocked by our spectral filter.

Comparing LIF intensity to derived temperature profiles (Fig. 6), one can see that fitting LIF spectra below a certain intensity threshold results in less precise and apparently increasing temperature values. These parts of the temperature profiles were excluded during postprocessing.

\subsection{Laser intensity}

The signal-to-noise ratio in two-dimensional LIF measurements is usually limited by photon noise, and a stronger signal generally improves the quality of LIF spectra. However, the A-X electronic transition of NO can be saturated with moderate laser power [12] due to its high oscillator strength. Figure 5 shows that NO LIF in Bunsen flame can be saturated even by the unfocused laser beam. For temperature measurements, we reduced the laser 


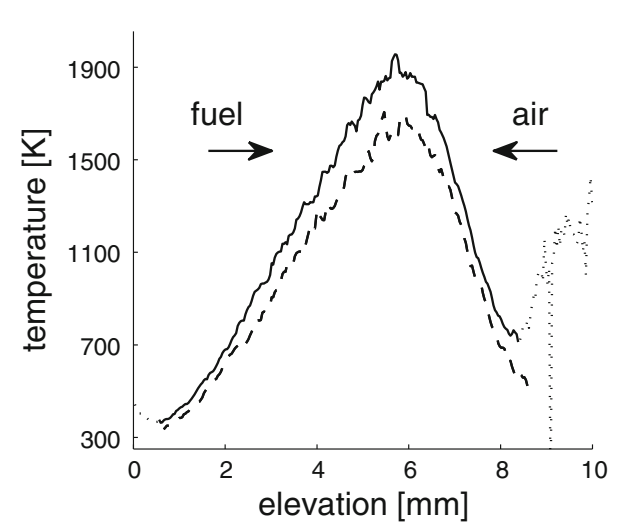

Fig. 6 Fitted temperature profiles for NO LIF spectra taken with maximal $(100 \%$, dashed line $)$ and reduced $(10 \%$, solid line ) laser energy fluence in methane/air flame. Dotted line shows the part of temperature fits that was discarded due to very low LIF intensity

fluence to about $20 \mathrm{~J} / \mathrm{m}^{2}$ that is in the range of linear relation of LIF intensity to laser power.

The main limiting factor for the linear excitation regime is the rate at which collisional quenching depopulates the excited state of nitric oxide [23]. This rate varies greatly depending on gas composition and temperature, and the limit on laser power will depend on the type of observed flame but also on a particular location within the flame. To understand how laser saturation could change the fitted temperature profiles, we compared measurements done with laser energy fluence of 200 and $20 \mathrm{~J} / \mathrm{m}^{2}$. Both scans were fitted with LIFSIM, producing temperature profiles as shown on Fig. 6. Measurements done without precautions against saturation resulted in $15 \%$ lower temperatures or as much as $250 \mathrm{~K}$ difference in the flame front region. Both fits converged well, and there was no indication of a systematic error. Without testing the effect of laser power on fitted temperatures, one can have large systematic error in measured temperature values that is impossible to correct, because it depends, among other things, on laser power distribution within the laser sheet.

\section{Results and discussion}

\subsection{Comparing temperature fits with the model}

To check the accuracy of the temperature fits, we compared them with results of chemical kinetic modeling. We used the Cantera software [24] to model axisymmetric, opposedflow diffusion flames using the GRI-Mech 3.0 kinetics mechanism [25]. Calculations were done for $300 \mathrm{~K}$ inlet temperature and 1 bar pressure with the distance between the gas inlets $9.5 \mathrm{~mm}$ (measured value) and the same gas mass flows as used in the measurements. Radiative losses are not included in this model, but it was shown [18] that in counterflow diffusion flames, they do not exceed $50 \mathrm{~K}$. Measurements and calculations are compared in Fig. 7.

The flame produced in the counterflow burner could be adequately represented with a one-dimensional axisymmetric model for a variety of fuels. Calculated and measured profiles match very well in position and absolute value, except for the hottest part where measured temperatures are consistently lower than predicted values (by as much as $150 \mathrm{~K}$ in propane flame). This difference is significant as the statistical fluctuation of measured temperature compared to smoothed profile was on the order of $40 \mathrm{~K}$. We discussed in chapter 3.1 that NO LIF spectrum in the hottest region generally fits to the Boltzmann energy distribution, and it is not clear if the observed deviation can be caused by a contribution of non-equilibrium NO to the spectrum.

Overall, good agreement between the measurement and the calculation signifies the suitability of the burner design, the measurement method and the model. While measured temperatures could be biased, the shape and position of the temperature peak is likely to be correct. On the other hand, Cantera calculation could predict the peak at the wrong place if, for example, specified mass flows did not match actual values. Gas-flow speed could also increase if the ceramic honeycombs at the gas inlets are heated by flame radiation. Auxiliary temperature measurements in a methane/air flame with $150 \mathrm{ppm}$ of NO seeded in both flows yield inlet temperatures of $300 \mathrm{~K}$ on the fuel side and $340 \mathrm{~K}$ on the air side. According to our calculations, such change in boundary conditions results in less than $20 \mathrm{~K}$ increase of the temperature peak and in a negligible shift of its position.

It was discussed in Sect. 3.1 that in regions of strong soot formation the NO LIF signal is strongly decreased due to absorption of laser light by soot precursors. Low signal intensity can result in unreliable temperature fits, as observed for ethylene flames (outlined region on Fig. 7d). The absorption in the propane flame is not as strong and does not influence the temperature fits (Fig. 7c). In particular, there is no temperature shift due to broadband PAH fluorescence. However, there is a strong discrepancy in the model at the fuel inlet where the derived temperature values fall below $200 \mathrm{~K}$. This effect, explicable in retrospective, was puzzling at first and led to additional investigations described in the next section.

\subsection{Collisional broadening and gas composition effects}

We took a LIF spectrum of nitric oxide in cold propane seeded with $100 \mathrm{ppm}$ of NO and compared it with corresponding spectrum taken in air (see Fig. 8). The width of NO rotational lines is defined by collisional broadening in 
Fig. 7 Fitted temperature profiles (solid line) compared with the model (dashed line). NO LIF Spectra were measured with a methane, $\mathbf{b}$ ethane, c propane and $\mathbf{d}$ ethylene as a fuel and air as an oxidant, both flow velocities set at $10 \mathrm{~cm} / \mathrm{s}$. Calculations for onedimensional axisymmetric diffusion flames were done with Cantera using GRI-Mech 3.0 mechanism. Large discrepancies marked with dotted ellipses are discussed in text
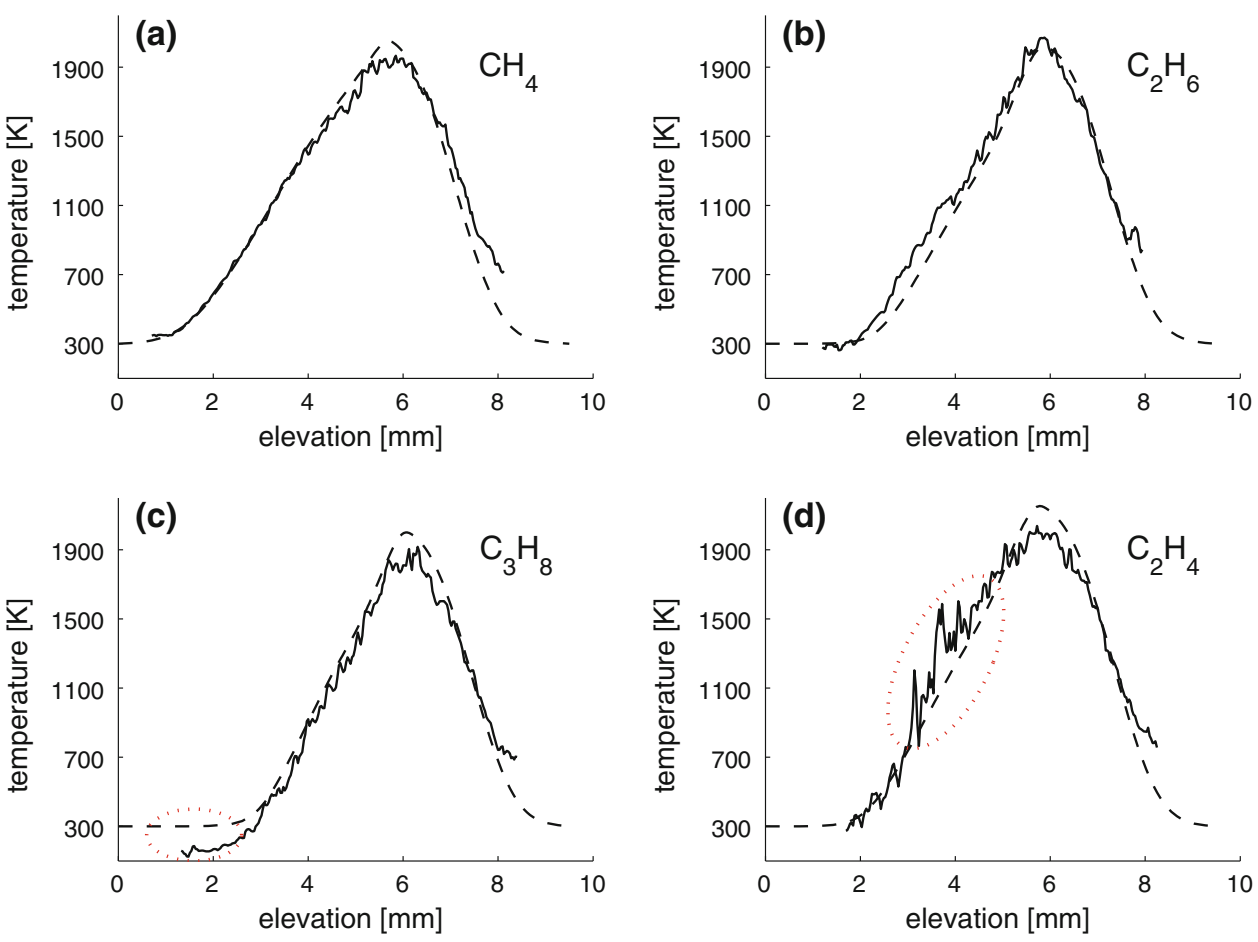

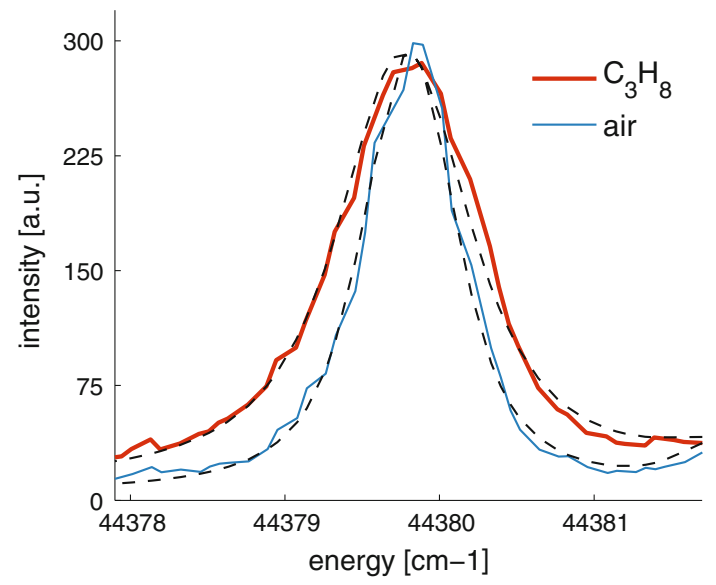

Fig. 8 NO LIF spectra taken at $300 \mathrm{~K}$ in propane (red) and air (blue), seeded with $100 \mathrm{ppm}$ of NO. Respective line fits by LIFSIM are shown with dashed lines

both cases, and collisions with the heavier propane molecules result in significantly broader lines. When nitrogen was assumed as the only collisional partner, spectral fits converged to much lower temperatures to compensate the lack of broadening (collisional broadening becomes stronger at lower temperatures as gas density increases). The value of the collisional broadening coefficient for propane has not been reported in literature yet. By fitting the spectrum taken in propane for $300 \mathrm{~K}$, we obtained a collisional linewidth of $2 \gamma=0.85 \mathrm{~cm}^{-1}$, which is $45 \%$ more than reported for nitrogen $0.585 \mathrm{~cm}^{-1}$ at the same conditions [26].

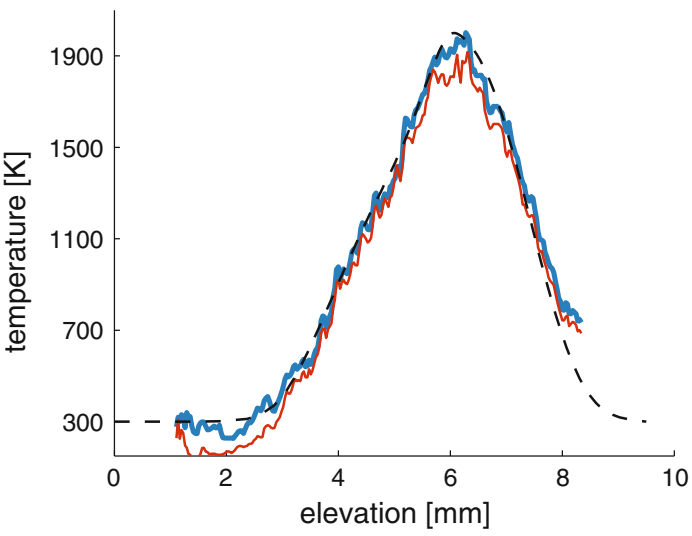

Fig. 9 Temperature profile of a propane/air flame, determined using specific gas composition: Blue line - detailed flame composition calculated with Cantera; red line - nitrogen. The calculated temperature profile from Cantera is shown with a dashed line

We used the results of the Cantera calculation of the propane-air flame to provide detailed temperature-dependent gas composition to the LIFSIM program. Since the collisional broadening coefficient of propane was determined only for one temperature, we approximated its temperature dependence with the same exponent as for nitrogen: $2 \gamma=(300 \mathrm{~K} / \mathrm{T})^{0.75} 0.85 \mathrm{~cm}^{-1} / \mathrm{atm}$. Figure 9 shows temperature fits with and without detailed information on gas composition and compares them with the Cantera calculation. The fit that takes the gas composition into account agrees much better with the model calculation with the temperature converging properly to $300 \mathrm{~K}$ at the 
fuel inlet. The difference between the two temperature fits decreases above $600 \mathrm{~K}$ as the collisional rate goes down, and the shape of the NO lines is determined by other factors such as laser linewidth and Doppler broadening. Therefore, there is no significant difference between two fits in the temperature range of $600-1,800 \mathrm{~K}$. We conclude that the NO spectra could be fitted adequately assuming nitrogen as the only collisional partner, but specifying the gas composition would improve the results.

\section{Conclusions}

A new counterflow burner was constructed and used to study opposed-flow diffusion flames. These flames were successfully modeled using the one-dimensional axisymmetric flame model of Cantera and the GRI-Mech 3.0 reaction mechanism.

The diffusion flames produced sufficient amounts of nitric oxide to record and fit NO LIF spectra in the region from 1.5 to $8 \mathrm{~mm}$ above the fuel inlet. Temperature profiles were determined with multiline NO LIF thermometry for a variety of sooting and non-sooting conditions and compared with calculations in Cantera. Excellent agreement confirms the accuracy of the method as well as its robustness toward light scattering, absorption and interfering fluorescence. However, care must be taken to limit the laser power as NO excitation deviates from the linear regime even at moderate laser power and that can lead to temperatures being underpredicted by as much as $15 \%$.

Good agreement with calculations was achieved even though detailed gas composition was neglected, and nitrogen was assumed as the only gas component. Propaneair flames were an exception as the collisional broadening coefficient of NO in propane is much larger than in nitrogen and should be taken into account for accurate temperature fitting whenever collisional broadening dominates NO line shapes-at temperatures below $600 \mathrm{~K}$ or at elevated pressures. We determined that the collisional broadening coefficient of $\mathrm{NO}$ in propane is $0.85 \mathrm{~cm}^{-1} / \mathrm{atm}$ at $300 \mathrm{~K}$.

Absorption of laser radiation in strongly sooting ethylene flames reduced the accuracy of the temperature fit. In other flames, the absorption was not as strong and did not affect the quality of fitted temperature profiles. We conclude that NO LIF multiline thermometry is well suited for low and moderately sooting diffusion flames, offering the possibility of truly non-intrusive temperature measurements that may be particularly important for soot-formation studies.

Acknowledgments This study is part of a project funded by Methanol Casale (www.casale.ch). We thank Dr. Wolfgang Kreutner for the design of the counterflow burner and Prof. Christof Schulz for a copy of LIFSIM software.

\section{References}

1. S. Will, S. Schraml, K. Bader, A. Leipertz, Performance characteristics of soot primary particle size measurements by timeresolved laser-induced incandescence. Appl. Opt. 37(24), 5647-5658 (1998)

2. S.P. Kearney, R.W. Schefer, S.J. Beresh, T.W. Grasser, Temperature imaging in nonpremixed flames by joint filtered Rayleigh and Raman scattering. Appl. Opt. 44(9), 1548-1558 (2005)

3. A.C. Eckbreth, Laser Diagnostics for Combustion Temperature and Species (Gordon and Breach Science Publishers, Amsterdam, 1996)

4. P.-E. Bengtsson, M. Aldén, S. Kröll, D. Nilsson, Vibrational CARS thermometry in sooty flames: quantitative evaluation of C2 absorption interference. Combust. Flame 82(2), 199-210 (1990)

5. F. Beyrau, T. Seeger, A. Malarski, A. Leipertz, Determination of temperatures and fuel/air ratios in an ethene-air flame by dualpump CARS. J. Raman Spectrosc. 34(12), 946-951 (2003)

6. C.F. Kaminski, J. Engström, M. Aldén, Quasi-instantaneous twodimensional temperature measurements in a spark ignition engine using 2-line atomic fluorescence. Symp. Int. Combust. 27(1), 85-93 (1998)

7. P.R. Medwell, Q.N. Chan, P.A.M. Kalt, Z.T. Alwahabi, B.B. Dally, G.J. Nathan, Development of temperature imaging using two-line atomic fluorescence. Appl. Opt. 48(6), 1237-1248 (2009)

8. J.M. Seitzman, R.K. Hanson, Two-line planar fluorescence for temporally resolved temperature imaging in a reacting supersonic flow over a body. Appl. Phys. B 57(6), 385-391 (1993)

9. M.P. Lee, B.K. McMillin, R.K. Hanson, Temperature measurements in gases by use of planar laser-induced fluorescence imaging of NO. Appl. Opt. 32(27), 5379-5396 (1993)

10. M.C. Drake, J.W. Ratcliffe, High-temperature quenching crosssections for nitric-oxide laser-induced fluorescence measurements. J. Chem. Phys. 98(5), 3850-3865 (1993)

11. A.O. Vyrodov, J. Heinze, M. Dillmann, U.E. Meier, W. Stricker, Laser-induced fluorescence thermometry and concentration measurements on NOA-X (0-0) transitions in the exhaust gas of high pressure CH4/air flames. Appl. Phys. B 61(5), 409-414 (1995)

12. W.G. Bessler, C. Schulz, Quantitative multi-line NO-LIF temperature imaging. Appl. Phys. B-Lasers Opt. 78(5), 519-533 (2004)

13. A. Khartabil, M. Leschowski, C. Hecht, T. Dreier, C. Schulz, Spatially and spectrally resolved chemiluminescence and temperature measurements in counterflow-diffusion flames (2009), in 4th European Combustion Meeting, Proceedings of Vienna University of Technology, 14th-17th April 2009

14. T. Lee, W.G. Bessler, H. Kronemayer, C. Schulz, J.B. Jeffries, Quantitative temperature measurements in high-pressure flames with multiline NO-LIF thermometry. Appl. Opt. 44(31), 6718-6728 (2005)

15. B. Atakan, A.T. Hartlieb, Laser diagnostics of NO reburning in fuel-rich propene flames. Appl. Phys. B 71(5), 697-702 (2000)

16. A.T. Hartlieb, B. Atakan, K. Kohse-Hoinghaus, Temperature measurement in fuel-rich non-sooting low-pressure hydrocarbon flames. Appl. Phys. B-Lasers Opt. 70(3), 435-445 (2000)

17. P. Glarborg, Hidden interactions-Trace species governing combustion and emissions. Proc. Combust. Inst. 31(1), 77-98 (2007) 
18. A. Beltrame, P. Porshnev, W. Merchan-Merchan, A. Saveliev, A. Fridman, L. Kennedy, O. Petrova, S. Zhdanok, F. Amouri, O. Charon, Soot and NO formation in methane-oxygen enriched diffusion flames. Combust. Flame 124(1-2), 295-310 (2001)

19. C.J. Sung, C.K. Law, Dominant chemistry and physical factors affecting no formation and control in oxy-fuel burning. Symp. Int. Combust. 27(1), 1411-1418 (1998)

20. S.V. Naik, N.M. Laurendeau, Quantitative laser-saturated fluorescence measurements of nitric oxide in counter-flow diffusion flames under sooting oxy-fuel conditions. Combust. Flame 129(1-2), 112-119 (2002)

21. H. Tsuji, Counterflow diffusion flames. Prog. Energy Combust. Sci. 8(2), 93-119 (1982)

22. W. Bessler, C. Schulz, V. Sick, J.W. Daily, A versatile modeling tool for nitric oxide LIF spectra, (Chicago, March 16-19, 2003, paper PI05, 2003)
23. J.W. Daily, W.G. Bessler, C. Schulz, V. Sick, T.B. Settersten, Nonstationary collisional dynamics in determining nitric oxide laser-induced fluorescence spectra. AIAA J. 43(3), 458-464 (2005)

24. D. Goodwin, Cantera: Object-Oriented Software for Reacting Flows. http://cantera.github.io/docs/sphinx/html/index.html

25. G.P. Smith, D.M. Golden, M. Frenklach, N.W. Moriarty, B. Eiteneer, M. Goldenberg, C.T. Bowman, R.K. Hanson, S. Song, W.C. Gardiner, V.V. Lissianski, Z. Qin, GRI-Mech 3.0, GRIMech 3.0. [Online]. Available: http://www.me.berkeley.edu/gri_ mech/

26. A.Y. Chang, M.D. DiRosa, R.K. Hanson, Temperature dependence of collision broadening and shift in the NO $\mathrm{A} \leftarrow \mathrm{X}(0,0)$ band in the presence of argon and nitrogen. J. Quant. Spectrosc. Radiat. Transf. 47(5), 375-390 (1992) 